\title{
Terahertz radiation from oscillating electrons in laser-induced wake fields
}

\author{
Li-Hua Cao, ${ }^{1, *}$ Wei Yu, ${ }^{2}$ Han Xu, ${ }^{2}$ Chun-Yang Zheng, ${ }^{1}$ Zhan-Jun Liu, ${ }^{1}$ Bin Li,${ }^{1}$ and A. Bogaerts ${ }^{3}$ \\ ${ }^{1}$ Institute of Applied Physics and Computational Mathematics, P.O. Box 8009, Beijing 100088, China \\ ${ }^{2}$ Shanghai Institute of Optics and Fine Mechanics, Shanghai 201800, China \\ ${ }^{3}$ Department of Chemistry, University of Antwerp, B-2610 Wilrijk-Antwerp, Belgium
}

(Received 24 March 2004; published 14 October 2004)

\begin{abstract}
Strong terahertz $\left(1 \mathrm{THz}=10^{12} \mathrm{~Hz}\right)$ radiation can be generated by the electron oscillation in fs-laser-induced wake fields. The interaction of a fs-laser pulse with a low-density plasma layer is studied in detail using numerical simulations. The spatial distribution and temporal evolution of terahertz electron current developed in a low-density plasma layer are presented, which enables us to calculate the intensity distribution of $\mathrm{THz}$ radiation. It is shown that laser and plasma parameters, such as laser intensity, pulse width, and background plasma density, are of key importance to the process. The optimum condition for wake-field excitation and terahertz emission is discussed upon the simulation results. Radiation peaked at $6.4 \mathrm{THz}$, with $900 \mathrm{fs}$ duration and $9 \%$ bandwidth, can be generated in a plasma of density $5 \times 10^{17} \mathrm{~cm}^{-3}$. It turns out that the maximum radiation intensity scales as $n_{0}{ }^{3} a_{0}{ }^{4}$ when wake field is resonantly excited, where $n_{0}$ and $a_{0}$ are, respectively, the plasma density and the normalized field amplitude of the laser pulse.
\end{abstract}

DOI: 10.1103/PhysRevE.70.046408

PACS number(s): 52.38.- r, 52.35.Mw, 52.27.Ny, 52.65.-y

\section{INTRODUCTION}

Terahertz radiation (or $\mathrm{T}$ rays), having a frequency between microwave and laser, has received considerable attention in recent years. As a nonionizing electromagnetic radiation with submillimeter wavelength, it has promising applications in biological imaging, surface chemistry, and high-field condensed matter studies $[1,2]$. Unlike microwave and laser, there are still no promising candidates for highefficiency T-ray generators, this "terahertz gap" greatly limits its applications [3]. Various methods, based on the principles of synchrotron radiation [4], transition radiation [5,6], and frequency up conversion of electromagnetic radiation [7], were proposed to generate terahertz radiation.

Laser pulse with femtosecond width is considered as a novel source of terahertz radiation since its pulse width is comparative with the wavelength of $\mathrm{T}$ rays. The fs-laser pulse can be used to resonantly excite terahertz electron oscillation, or terahertz electron current, in a low-density plasma. If the laser pulse width and plasma density are properly chosen to meet the resonant condition for wake-field excitation, strong electron current is generated in plasma, which can emit $\mathrm{THz}$ radiation to the surrounding space. $\mathrm{THz}$ emission from laser excited plasma wave has received considerable attention. The first experiment was reported earlier [8] and the radiation from the vacuum-plasma interface was investigated recently by particle-in-cell (PIC) simulations [9]. However, there has been no detailed description on distant $\mathrm{THz}$ radiation and the low frequency radiation by the oscillating electrons in laser-induced wake field is not well understood.

In this paper, two-dimensional particle-in-cell simulations are presented for the interaction of a fs-laser pulse with a low-density plasma. The spatial distribution and temporal

*Email address: cao_lihua@iapcm.ac.cn evolution of terahertz electron current developed in the plasma layer are studied in detail. A well-established formula for radiation is then applied to calculate the intensity of $\mathrm{THz}$ radiation. Strong terahertz emission has been observed in the simulations. It is shown that the laser and plasma parameters, such as laser intensity, pulse width, and plasma density, are of key importance to the process. The optimum condition for wake-field excitation and T-ray emission is discussed upon the simulation results.

\section{THz RADIATION INDUCED BY WAKE EXCITATION}

Terahertz radiation from laser-irradiated plasmas is attributed to the resonant excitation of terahertz electron oscillations in plasmas when the plasma density happens to match the laser pulse width. When a laser pulse propagates through a plasma, the displacement of plasma electrons driven by the ponderomotive force of laser pulse results in a wake plasma wave, in which the electrons oscillate at the plasma frequency $\omega_{p}$, where $\omega_{p}=\left(4 \pi n_{0} e^{2} / m\right)^{1 / 2}, e$ and $m$ are, respectively, the electron charge and rest mass. The oscillating electrons create regions of net positive and negative charge, which in turn forms an electrostatic wake field that propagates with phase velocity set by the laser group velocity

$$
V_{\mathrm{ph}}^{\mathrm{wake}}=v_{g}=c\left(1-\omega_{p}{ }^{2} / \omega_{0}{ }^{2}\right)^{1 / 2},
$$

where $\omega_{0}$ is the laser frequency and $c$ is the speed of light in vacuum. By an appropriate choice of the laser and plasma parameters, wake-field and wake-plasma waves can be effectively generated behind the laser pulse with a highly effective manner. According to the standard laser wake field theory $[10,11]$, in the nonrelativistic regime the optimum wake-field excitation occurs when the laser pulse width equals to half a plasma wavelength. A large amplitude longitudinal plasma wave can be resonantly excited and the peak electric field is given by [10] 


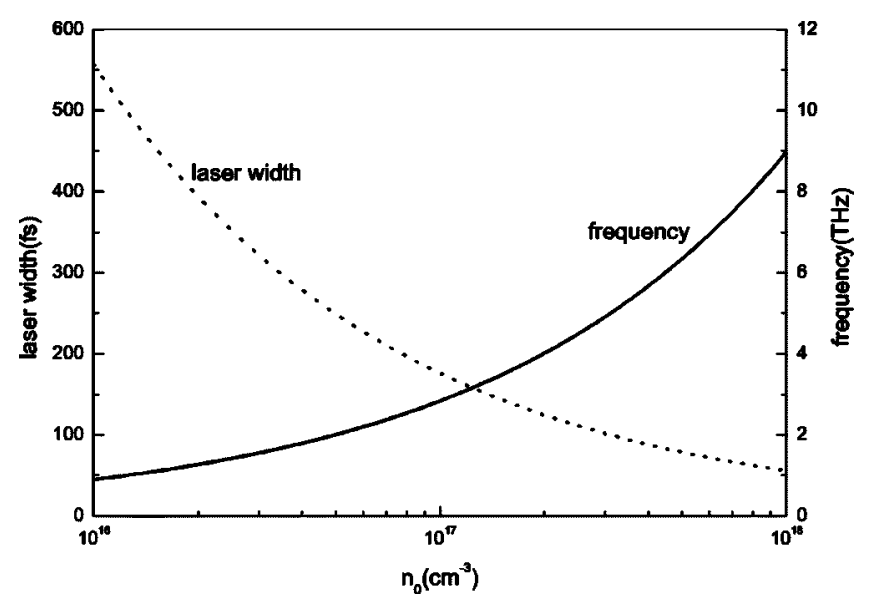

FIG. 1. The frequency of T ray (solid line) and resonant laser width (dashed line) depending on the electron density. THz radiations in the $1-10 \mathrm{THz}$ frequency range can be generated in the plasma densities $10^{16}-10^{18} \mathrm{~cm}^{-3}$ with laser widths at range $50-500$ fs.

$$
E_{x} \approx \frac{\pi^{2} m c^{2}}{e} \frac{a_{0}^{2}}{4 \lambda_{p}},
$$

where $\lambda_{p}=2 \pi c / \omega_{p}$ is the plasma wavelength.

Inside the laser pulse, electrons are accelerated by the ponderomotive forces arising from the high gradients of the laser intensity profile in the ascending front of the laser pulse and decelerated in the descending front. The resulting electron displacement leads to a strong charge separation field, which in turn tends to restrict the motion of the displaced electrons. After the laser pulse passes, a large space charge field remains and its relaxation leads to wake-plasma oscillations.

The powerful terahertz electron current associated with the wake plasma oscillation enables the plasma to emit electromagnetic radiation at $\mathrm{THz}$ frequencies. The frequency of the $\mathrm{T}$ ray thus generated is given by

$$
f=c / \lambda_{p}=8.972 \times 10^{-9} \sqrt{n_{0}\left(\mathrm{~cm}^{-3}\right)}(\mathrm{THz}),
$$

which depends on the plasma density and is independent of laser parameters. To obtain radiation in the $1-10 \mathrm{THz}$ range, the corresponding plasma density should be in the range of $10^{16}-10^{18} \mathrm{~cm}^{-3}$. From the condition for wake-field excitation $L \approx \lambda_{p} / 2$, we get the laser pulse width at resonance

$$
L \approx 0.5 \lambda_{p} / c=5.57 \times 10^{10} / \sqrt{n_{0}\left(\mathrm{~cm}^{-3}\right)} \text { (fs) } .
$$

The dependence of the frequency of terahertz emission and the resonant pulse width on plasma density is plotted in Fig. 1. The optimum laser pulse duration for T-ray generation through wake excitation is in the range of 50-500 fs.

\section{RESULTS OF PIC SIMULATIONS}

In order to get more insight into $\mathrm{THz}$ radiation induced by wake excitation, numerical simulations are performed with a two-dimensional (2D) PIC code. In the simulations, Gaussian laser pulses with wavelength $\lambda=1 \mu \mathrm{m}$ (the correspond-

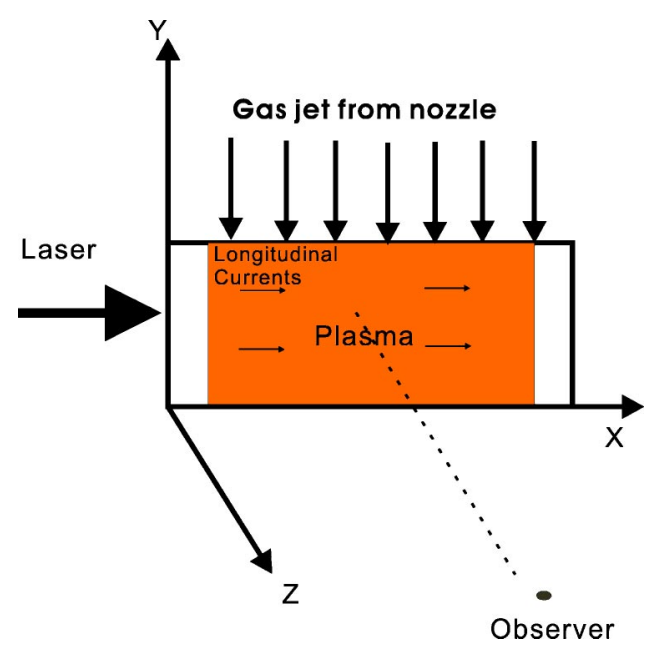

FIG. 2. Scheme of the simulation geometry of two dimensional PIC code.

ing laser period $T \sim 3.3 \mathrm{fs}$ ), spot size $10 \mu \mathrm{m}$, and pulse duration 50-500 fs are launched into homogeneous plasmas with density $10^{16}-10^{18} \mathrm{~cm}^{-3}$. The laser peak amplitude is $a_{0}$ $\sim 0.59$, which is still below the threshold for relativistic effect. The two-dimensional simulation box is $100 \lambda$ long and $50 \lambda$ in width, the plasma layer is $80 \lambda$ in length, with two vacuum gaps of $10 \lambda$ assigned at the left and right boundaries respectively, as schemed in Fig. 2. Experimentally, the plasma layer can be produced by irradiating a laser pulse onto a gas jet that streams from a nozzle in the $y$ direction. The laser propagates in the $x$ direction and the $\mathrm{THz}$ radiation is then measured in the $z$ direction. This configuration is helpful to reduce the absorption of $\mathrm{T}$ rays by plasma.

The initial velocities of plasma electrons and ions are assumed to be in Maxwellian distributions, with temperatures of $300 \mathrm{eV}$ for electrons and $100 \mathrm{eV}$ for ions. We use a spatial mesh of $1024 \times 512$ cells with $10^{7}$ electrons and the same for the ions, they initially fill the simulation box with a uniform density profile and can move in the $x-y$ plane. The ions are protons with mass $M=1836 \mathrm{~m}$ and $Z=1$. The simulations continue for a longer time even after the laser pulse leaves the box.

The following are the simulation results obtained in a homogeneous underdense plasma with density $5 \times 10^{-4} n_{c} \approx 5$ $\times 10^{17} \mathrm{~cm}^{-3}$, where $n_{c}$ is the critical density of the incidence pulse, the corresponding plasma wavelength is $44.72 \lambda$, the laser pulse width is chosen as $22.36 T \approx 74 \mathrm{fs}$, which is the optimum width for wake generation. The wake electric field is longitudinal, i.e., pointing to the direction of laser propagation, as shown by the spatial distributions of the largeamplitude electrostatic field for $t / T \sim 99$ and 198 [Figs. 3(a) and $3(\mathrm{~b})]$. Note that due to the finite spot size of laser beam [12], there is a laser electric field component in the region of $x>75 \lambda$ in the direction of laser propagation, as shown in Fig. 3(a), which has little contribution to electron energy gain. A longitudinal electrostatic plasma wave is efficiently driven behind the laser pulse since the resonant condition is satisfied. The normalized amplitude $e E_{x} / m \omega_{0} c$ of the wake field is about 0.004 from our simulations, compared with 0.003 estimated from Eq. (2), the corresponding electrostatic 
(a)

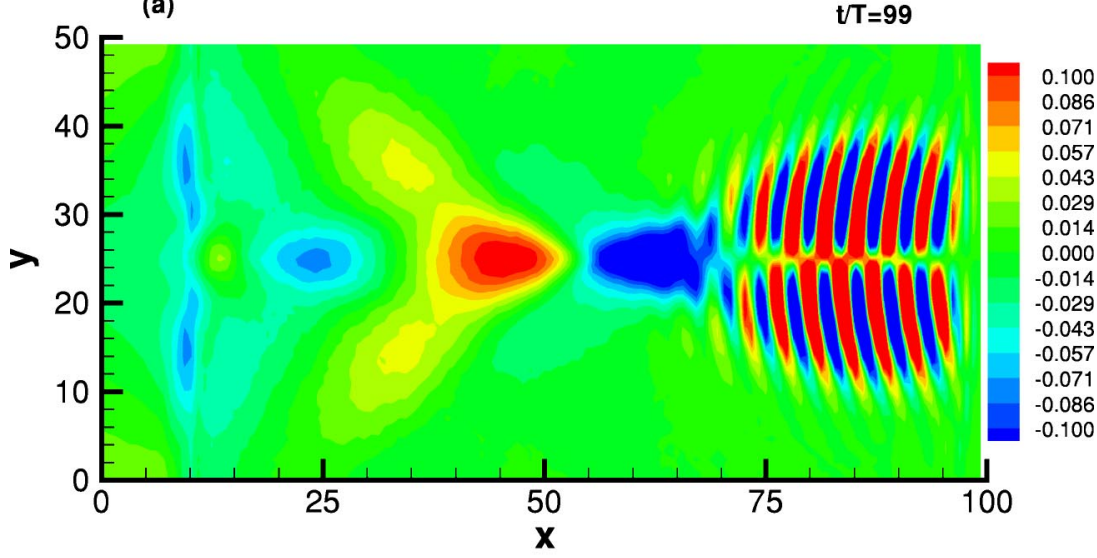

(b)

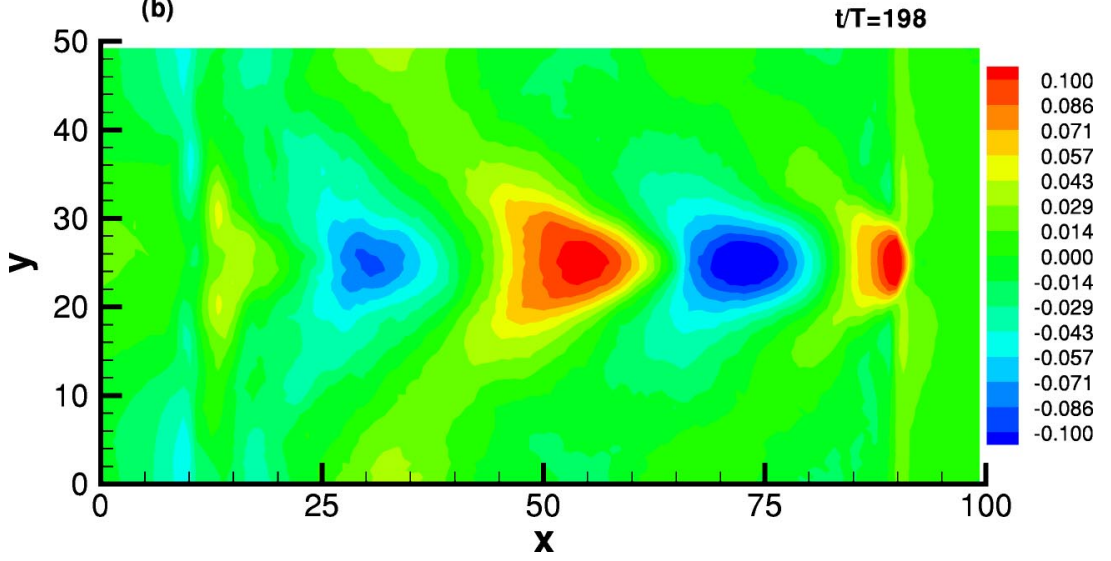

FIG. 3. Snapshots of the spatial configurations of normalized longitudinal electric field at $t / T=99$ (a) and 198 (b). field is of the order of $10^{8} \mathrm{~V} / \mathrm{cm}$, and the characteristic length of this low-frequency oscillation is close to plasma wavelength. Driven by the field, the electrons are accelerated and decelerated, the contour plot of longitudinal momentum is shown in Fig. 4. The electrons inside the laser pulse $(x>75 \lambda)$ undergo high-frequency quiver motion and have no contribution to $\mathrm{THz}$ radiation. On the other hand, the electrons inside laser-excited wake field move at a frequency of terahertz range and their kinetic energies are up to hundreds keV.

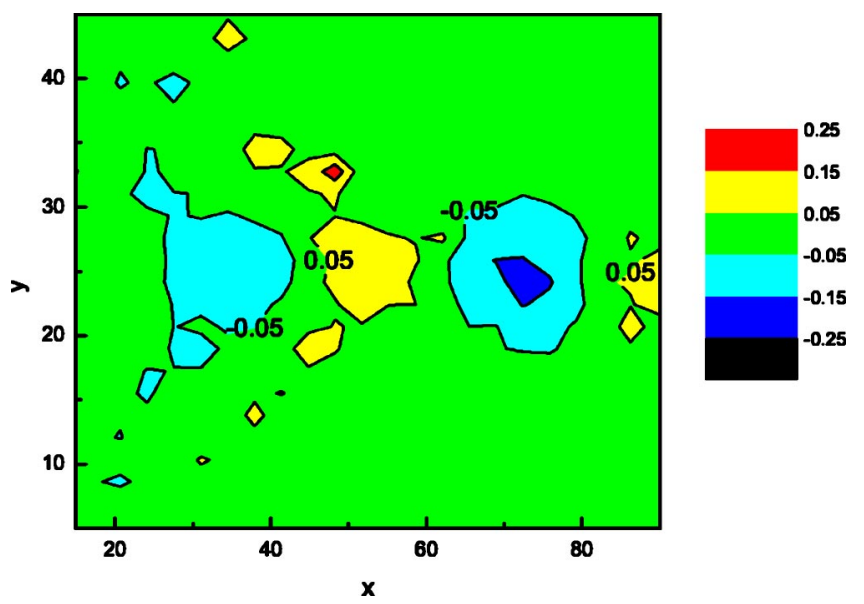

FIG. 4. The contour plot of electron longitudinal momentum at $t / T=99$, which is normalized by mc.
The longitudinal current created by the oscillating electrostatic field at $t / T=99$ and 198 are illustrated in Figs. 5(a) and $5(\mathrm{~b})$, the spatial period of the current is again the plasma wavelength. Note that the large-amplitude electrostatic field, oscillating electrons, and the longitudinal current can last for a longer time even after the laser pulse passes through the plasma layer. Both the transversal and longitudinal current densities at the center of the simulation box are tracked in each time step and the Fourier transforms are carried out, with the temporal and frequency spectra presented in Figs 6 and 7 , respectively. The transversal current is induced directly by the laser field, with a dominant spectral component at laser frequency and very little contribution in the terahertz regime, as exhibited in Fig. 6(b). On the other hand, the longitudinal current appears as soon as the laser pulse passes by, and lasts over for a time much longer than laser duration. Its frequency spectrum contains a strong terahertz component peaked at $6.4 \mathrm{THz}$ (i.e., the plasma frequency). It is evident that the $\mathrm{THz}$ emission originates from the longitudinal current. The maximum amplitude of the terahertz current density, normalized as $e J_{x} / m v_{\text {th }} \omega_{0}^{2}$, is roughly $6.37 \times 10^{-4}$ at t/T 93 as shown in Fig. 7(a), which correspond to a current density of $9 \times 10^{8} \mathrm{~A} / \mathrm{cm}^{2}$, with electron temperature $0.3 \mathrm{keV}$ or electron thermal velocity $v_{\text {th }} \sim 0.024 c$. The maximum current will be estimated to be in the order of $10^{3}$ A for a $10 \mu \mathrm{m}$ spot size.

From the temporal evolution of the longitudinal current density presented in Fig. 7(b), the current decays to $1 / e$ of its maximum value after 272 laser periods, i.e., the $\mathrm{THz}$ current 
(a)

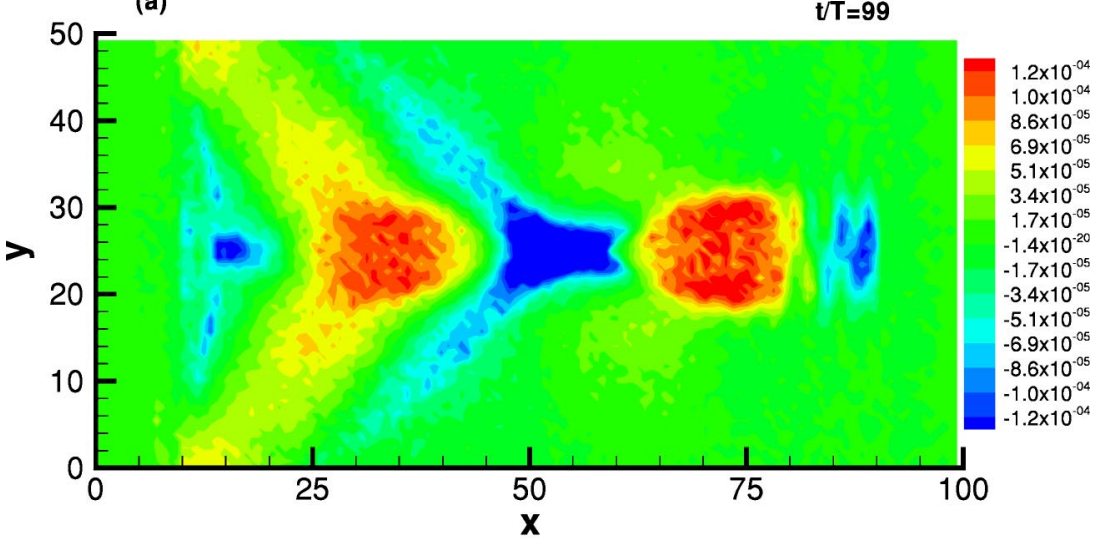

(b)

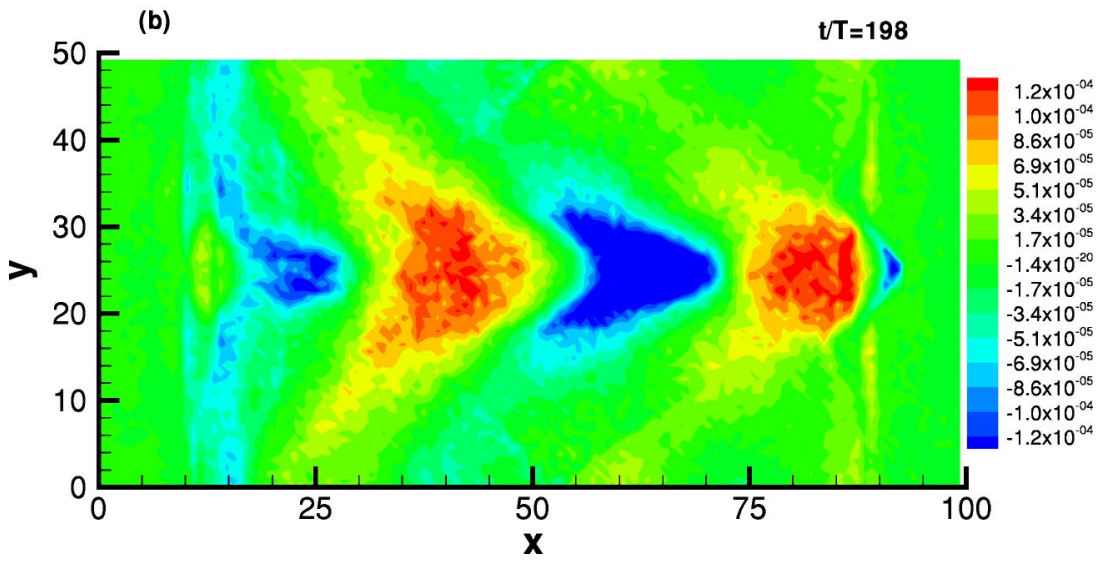

FIG. 5. Snapshots of the spatial distributions of longitudinal current densities at $t / T=99$ (a) and 198 (b). The current density is normalized by $m v_{\text {th }} \omega_{0}^{2} / e$, where $v_{\text {th }}=0.024 c$. and radiation can remain in plasma over about $900 \mathrm{fs}$, while the laser pulse escapes the simulation box at around $300 \mathrm{fs}$. The duration of the emission is quantitative agreement with the experiment measurement [8]. The weak damping of the plasma oscillation is due to the fact that electron-ion collision is less important on a time scale of hundreds femtosec- onds. The pulse duration of $\mathrm{THz}$ radiation relies on the attenuation rate of the terahertz current.

The radiated intensity of the terahertz radiation can be deduced from the temporal and spatial distributions of the longitudinal current. Here the far-field approximation is introduced since the observer is always far away from the cur-

$(L x / 2, L y / 2)$
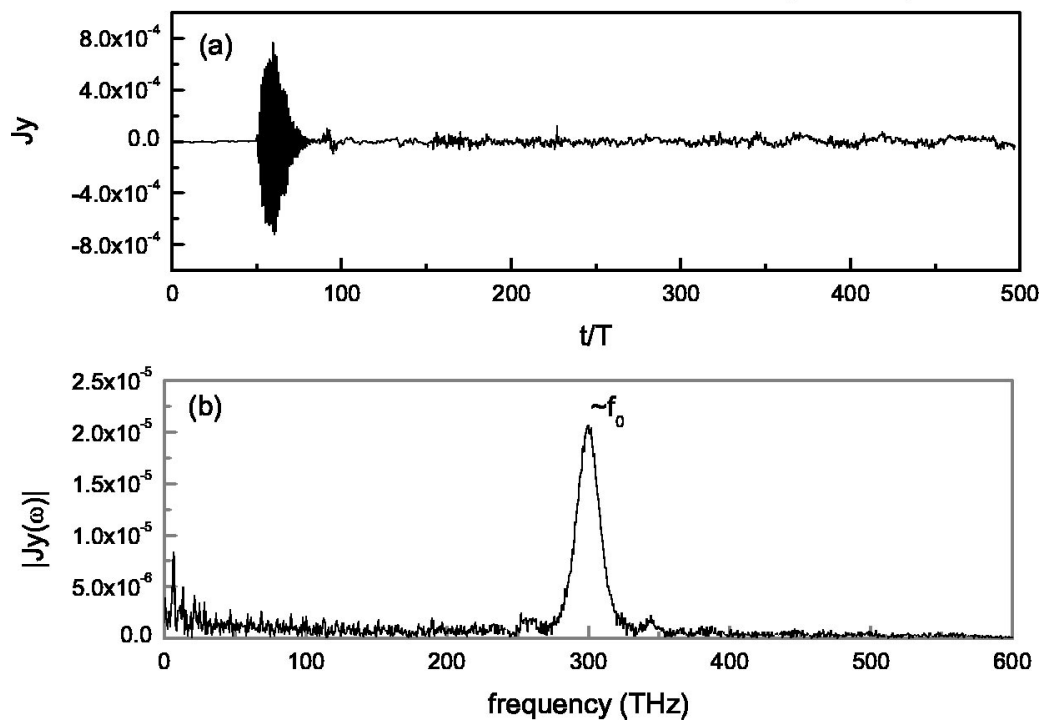

FIG. 6. Temporal evolution of the normalized transversal current density $\left(J_{y}\right)$ at the center of simulation box (a) and its Fourier transform (b). 

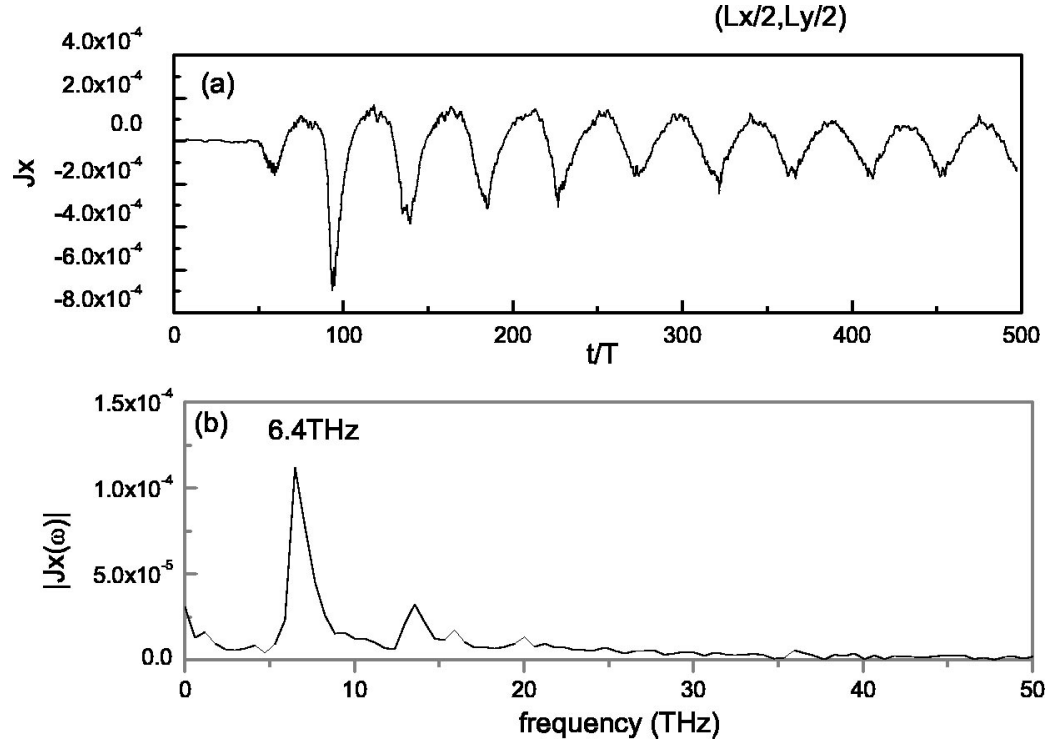

FIG. 7. Same as Fig. 6, but for the longitudinal current density $J_{x}$. rent. So the energy radiated per unit solid angle and per unit frequency interval from the current density distribution $j(\mathbf{r}, t)$ can be calculated by [13]

$$
\frac{d^{2} I}{d \omega d \Omega} \approx \frac{\omega^{2} \sin ^{2} \theta}{4 \pi^{2} c^{3}}\left|\int d t e^{i \omega t} J(t)\right|^{2},
$$

where $\theta$ is the angle of observation with respect to the laser propagation direction and $\omega$ is the angular frequency of the radiation, and $J(t)=\int d V j(\mathbf{r}, t)$. The time integration $\int d t e^{i \omega t} J(t)$ is the Fourier transform of the current density. Considering the T-ray emission in the direction perpendicular to the laser propagation, i.e., $\theta \sim \pi / 2$, then the intensity distribution becomes

$$
\frac{d^{2} I}{d \omega d \Omega} \approx \frac{\omega^{2}}{4 \pi^{2} c^{3}}\left|\int d t e^{i \omega t} J(t)\right|^{2} .
$$

We have performed additional diagnosis to investigate the nature of the radiation by recording the temporal and spatial evolution of longitudinal current density $j_{x}$ and then calculating numerically the radiation intensity from the formula (6). Figure 8 shows the spectrum of $\mathrm{THz}$ radiation observed at $\theta \sim \pi / 2$, a single broad peak centered at $6.4 \mathrm{THz}$ is clearly seen, as expected from the frequency spectrum of longitudinal current in Fig. 7(b), its full-width-at-half-maximum bandwidth $\Delta \omega / \omega$ is about 0.09 . Here only the longitudinal current density has to be accounted for, since the transversal current has very little contribution to $\mathrm{THz}$ radiation, as verified by the frequency spectra of $J_{x}$ and $J_{y}$ in Figs. 6 and 7.

In order to examine the optimum condition for T-ray emission, more simulations are conducted for different laser and plasma parameters. The resulting intensity spectra are summarized in Figs. 9 and 10. For the fixed plasma parameters, the strongest enhancement in $\mathrm{THz}$ radiation is achieved when the resonant condition for wake excitation is satisfied, i.e., when the laser pulse width equals half the plasma wavelength. The departures from the optimum laser pulse width will lead to weaker emission and larger of frequency band- width, compared with the resonance-case, as shown in Fig. 9(a). This indicates the importance of a proper choice of laser and plasma parameters.

Figure 9(b) gives the frequency spectra of radiation for different plasma densities, where the laser pulse widths are properly chosen to meet the resonant condition for wake excitation. Each curve has a single peak, the peak frequency varies with plasma density and is close to the corresponding plasma frequency as expected from Eq. (3). Since the amplitude of wake field is correlated to the plasma density through Eq. (2), the electrostatic field increases with increasing plasma density as $E_{x} \sim n_{0}{ }^{1 / 2}$. Providing the resonant condition for wake excitation is always met, higher plasma density results in higher peak radiated frequency and power, with the bandwidth remains almost unchanged. These results agree with that obtained by Hamster et al. [8].

Furthermore, it is instructive to evaluate the dependence of peak intensity of $\mathrm{THz}$ radiation on plasma density and laser intensity. For simplicity we consider the nonrelativistic

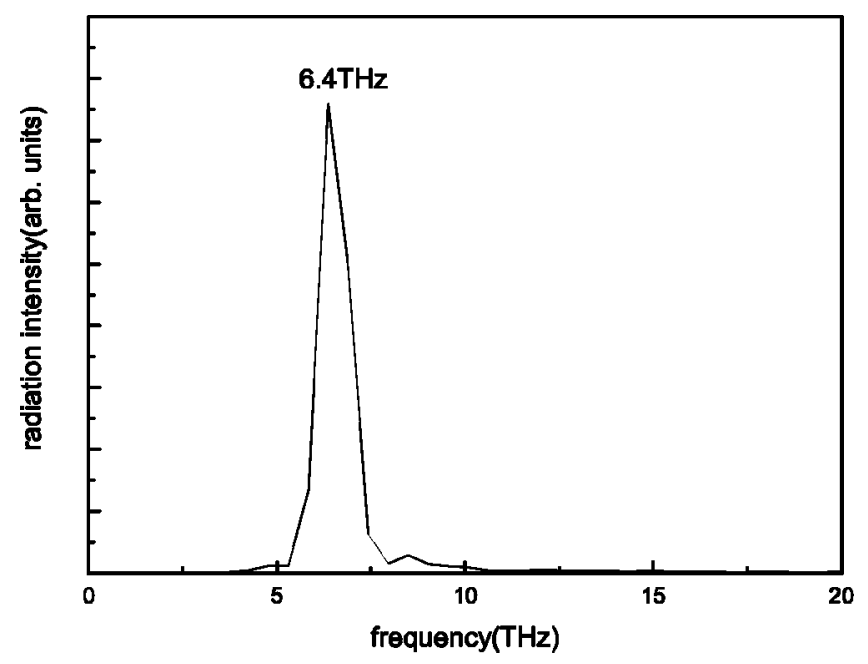

FIG. 8. The radiation intensity distribution as a function of frequency, the strongest excitation at frequency $6.4 \mathrm{THz}$ with $9 \%$ bandwidth is observed. 

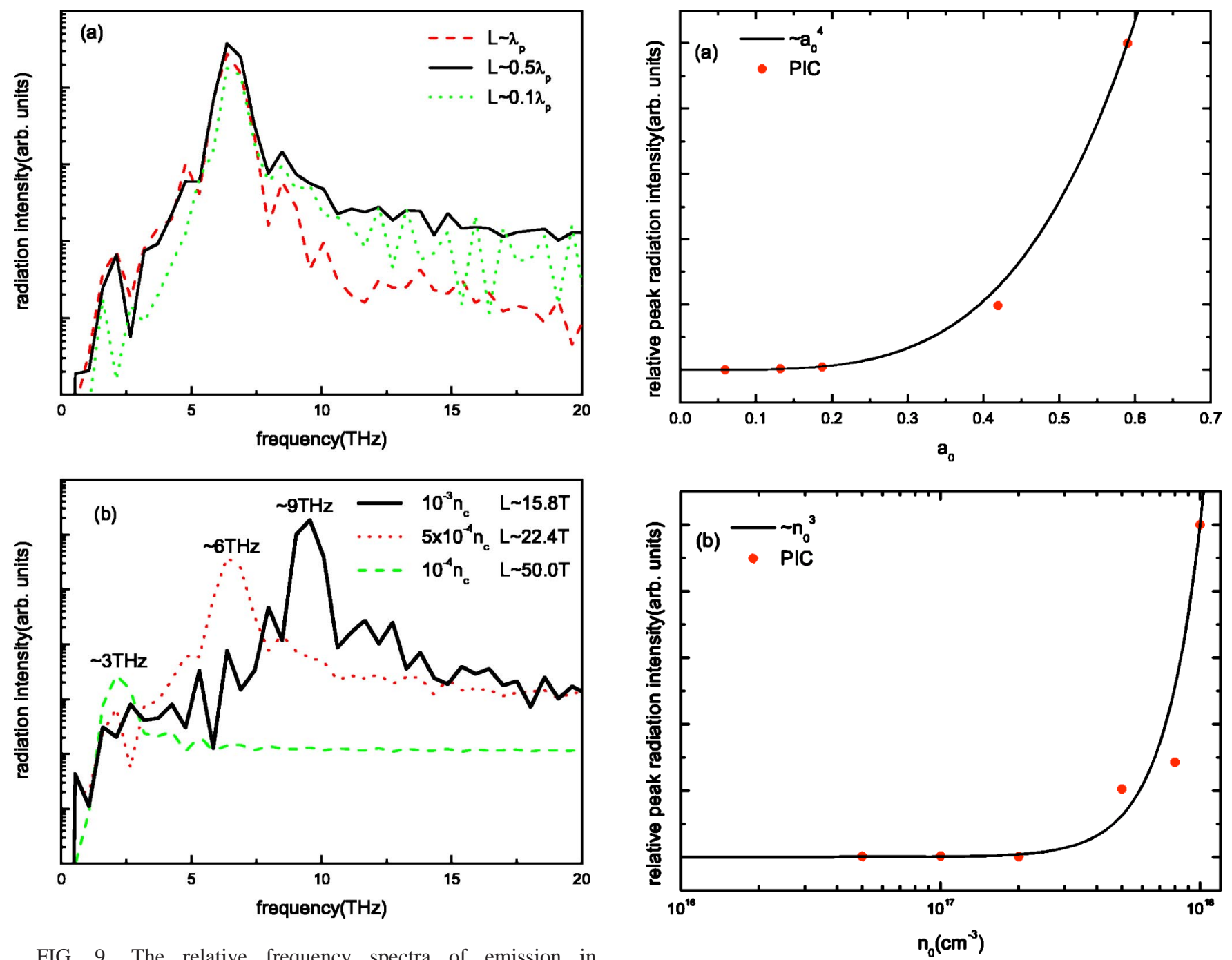

FIG. 9. The relative frequency spectra of emission in fixed plasma density $5 \times 10^{17} \mathrm{~cm}^{-3}$ for different laser widths $\quad L \sim 0.5 \lambda_{p}$ (solid line), $\quad L \sim \lambda_{p}$ (dashed line), and $L \sim 0.1 \lambda_{p}$ (dotted line) (a) in different density plasmas with the optimum laser widths: $n_{e}=10^{-3} n_{c}, \quad L \sim 15.8 \mathrm{~T}$ (solid line), $n_{e}=5 \times 10^{-4} n_{c}, \quad L \sim 22.4 \mathrm{~T}$ (dotted line) and $n_{e}=10^{-4} n_{c}$, $L \sim 50.0 \mathrm{~T}$ (dashed line) (b).

electron motion driven by the wake field $E_{x}$, time dependence of $\exp \left(-i \omega_{p} t\right)$ is assumed for the field and the longitudinal velocity $v_{x} \propto E_{x} / \omega_{p}$, so the radiation intensity is then given by

$$
\frac{d^{2} I}{d \omega d \Omega} \approx \frac{\omega^{2}}{4 \pi^{2} c^{3}}\left|\int d t e^{i \omega t} J_{x}(t)\right|^{2} \propto \omega^{2} n_{0}{ }^{2}\left|\nu_{x}\right|^{2} \propto \omega^{2} n_{0}{ }^{2} \frac{\left|E_{x}\right|^{2}}{\omega_{p}{ }^{2}} .
$$

Note that the maximum radiated frequency is close to the plasma frequency, by using the expression for peak electric field (2) we get

$$
\frac{d^{2} I}{d \omega d \Omega} \propto n_{0}{ }^{3} a_{0}{ }^{4} .
$$

The dependences of the peak radiation intensity on $n_{0}$ and $a_{0}$ are exhibited in Figs. 10(a) and 10(b), respectively, where the

FIG. 10. Scaling laws of peak radiation intensities with electron density $\left(\sim n_{0}{ }^{3}\right)$ (a) and laser intensity $\left(\sim a_{0}^{4}\right)$ in plasma of $5 \times 10^{17} \mathrm{~cm}^{-3}$ (b). The real lines represent the analytical scaling laws and the circle points show the PIC simulations at the optimum laser pulse widths.

solid lines are calculated from Eq. (8) and the circle points are the results of PIC simulations at the optimum laser width $L \approx \lambda_{p} / 2$. Both are basically in agreement.

However, very high plasma density is not appropriate since the radiated frequency depends on the plasma density through $f \propto \sqrt{n_{0}}$, if the density is too high the radiation emitted is no longer in terahertz range. It should be indicated that our study is confined to nonrelativistic regime (i.e., $a_{0}<1$ ), for ultraintense lasers with $a_{0}>1$, the electron motion and radiation are quite different: the electron oscillation is no longer sinusoidal and the radiated frequency consists of not only the frequency of electron oscillation but also its high harmonics. Meanwhile, the optimum pulse width becomes smaller as the laser intensity increases [14]. It is therefore very unlikely that higher plasma density and laser intensity can lead to stronger terahertz radiation. 


\section{CONCLUSIONS}

It is demonstrated via PIC simulations that a laserirradiated plasma has sufficient feasibility as a novel source of $1-10 \mathrm{THz}$ radiation, with plasma densities $10^{16}-10^{18} \mathrm{~cm}^{-3}$ and laser durations $50-500$ fs. Strong transient phenomena will enable the intense laser pulse to excite a large amplitude plasma wave, if the resonant condition is met. Strong longitudinal current at terahertz frequencies is observed by the simulations, which in turn results in the emission of pulsed $\mathrm{THz}$ radiation. The frequency of the radiation depends on the plasma density and its pulse duration is determined by the lifetime of the longitudinal current. In order to optimize the terahertz radiation, the resonant condi- tion for wake excitation has to be satisfied, otherwise, there will be a significant decrease in the peak radiation intensity and increase in the frequency bandwidth. If the laser pulse width is properly chosen to meet the resonant condition, the peak radiation intensity scales as $\sim n_{0}{ }^{3} a_{0}{ }^{4}$.

\section{ACKNOWLEDGMENTS}

One of the authors (L.-H. C.) would like to thank Professor Jinghong Li for his useful discussions. This work was supported by National High Technology ICF Committee of China and National Natural Science Fund of China (Grant Nos. 10335020, 10375011).
[1] D. M. Mittleman et al., Appl. Phys. B: Lasers Opt. 68, 1085 (1999).

[2] J. Orenstein and A. J. Millis, Science 288, 468 (2000).

[3] A. Corchis et al., Phys. Rev. B 64, 205204 (2004).

[4] T. Nakazato et al., Phys. Rev. Lett. 63, 1245 (1989).

[5] U. Happek, A. J. Sievers, and E. B. Blum, Phys. Rev. Lett. 67, 2962 (1991).

[6] W. P. Leemans et al., Phys. Rev. Lett. 91, 074802 (2003).

[7] R. L. Savage, C. Joshi, and W. B. Mori, Phys. Rev. Lett. 68, 946 (1992).

[8] H. Hamster, A. Sullivan, S. Gordon, W. White, and R. W.
Falcone, Phys. Rev. Lett. 71, 2725 (1993).

[9] Zheng-Ming Sheng, Hui-Chun Wu, Kun Li, and Jie Zhang, Phys. Rev. E 69, 025401(R) (2004).

[10] J. Krall, A. Ting, E. Esarey, and P. Sprangle, Phys. Rev. E 48, 2157 (1993).

[11] A. Pukhov, Rep. Prog. Phys. 66, 47 (2003).

[12] Y. I. Salamin and C. H. Keitel, Phys. Rev. Lett. 88, 095005 (2002).

[13] J. D. Jackson, Classical Electrodynamics, 3rd ed. (Wiley, New York, 1999), Chap. 14.

[14] M. Y. Yu et al., Phys. Plasmas 10, 2468 (2003). 